\title{
RESEARCH ON RADAR TARGETS RECOGNITION BY EXTRACTING 3-D CHARACTERISTIC FROM ISAR IMAGES
}

\author{
Liu, Feng and $\mathrm{Xu}$, Jiadong \\ Electronic Engineering Department, Northwestern Polytechnical University, Xi'an 710072 \\ E-mail:Beaconliu@263.net,Tel: (86)029-88491414
}

\begin{abstract}
Based on ISAR imaging technology and Computer Vision theory, a novel target recognition method extracting the 3-Dimensional characteristic of targets from ISAR images sequence is presented in this paper, which can get higher recognition rate with fewer samples.
\end{abstract}

Key words: ISAR, Scattering Centers, 3-Dimensional Characteristic, Target Identification

\section{INTRODUCTION}

With the developing of modern radar, a considerable amount of effort has been devoted to radar target identification [1]. Currently, most past work mainly focus on identifying targets with the one-dimensional feature of radar echo [2-3], which need a large numbers of training samples [2-3]. However, just as the 3-dimesional structure of a object could be estimated by its images shooting at different angles [4], similarly, the 3-D structure characteristic of the scattering centers of motion target can be determined through their ISAR (Inverse Synthetic Aperture Radar) image sequence. Furthermore, the target's 3-D structure characteristic is possible to be used to identify different targets. In this paper, we present the method to extract the radar target's 3-D structure parameters from the target's ISAR image sequence, and the experimental identification results for simulated targets. 


\section{ABOUT ISAR IMAGE SEQUENCE}

The theory and approach of the ISAR imaging are depicted in [5-6].

If the processing of ISAR imaging is constantly done several times for a dynamic target, the target's ISAR image sequence could be gained. An actual target's ISAR image sequence given in [6] is shown here in Figurel.
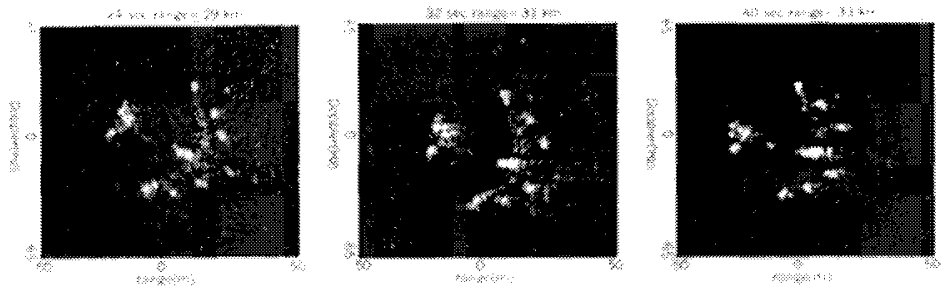

Figure l. Actual target's ISAR image sequence

\section{THE METHOD OF EXTRACTING 3-D CHARACTERISTIC FROM ISAR SEQUENCE}

The geometrical structure of the main scattering centers that distinctly illuminates the characteristic of a target. It is shown in Fig.1, that the information of the target's scattering centers included in the target's ISAR images is displayed as discrete spot cells distributing in different grayscale and area on the images. Conveniently, in this paper, the spot cell is defined as "Imaged Cell of Scattering Center (ICSC). In order to extract the 3-D structure of the target's scattering centers, the method of Optical Flow Estimation in Computer Vision field [7] is applied in the paper.

\subsection{Determining and tracking CICSC}

As to compute the ICSC's optical flow, firstly, the Centroid of Imaged Cell of Scattering Center (CICSC) should be determined and tracked to match the ICSCs between the frames. CICSC defined as the average point in an ICSC according to the grayscale (intensity of echo). In order to predict and track the moving trace of the ICSC between frames, the filtering model is applied as follows:

$$
X(k+1)=\left[\begin{array}{cccc}
1 & T & 0 & 0 \\
0 & 1 & 0 & 0 \\
1 & 0 & 0 & 0 \\
0 & 0 & 0 & 0
\end{array}\right] \boldsymbol{X}(k+1)+\left[\begin{array}{cc}
T^{2} / 2 & 0 \\
T & 0 \\
0 & 0 \\
0 & 0
\end{array}\right]\left[\begin{array}{l}
W_{1}(k) \\
W_{2}(k)
\end{array}\right]
$$




$$
\boldsymbol{Z}(k)=\left[\begin{array}{cccc}
1 & 0 & 0 & 0 \\
1 & 0 & -1 & 0
\end{array}\right] \boldsymbol{X}(k)+\left[\begin{array}{l}
V_{1}(k) \\
V_{2}(k)
\end{array}\right]
$$

where $\boldsymbol{X}(k)$ is the position state composed of the centroid position, the centroid offset, and the ecntroid position of last frame; $\boldsymbol{Z}(k)$ is composed of the measured values of the centroid position and offset; $\boldsymbol{W}$ and $\boldsymbol{V}$ is the noise; $T$ is the interval between frames. From (1), the motion and position parameters of CCICS in the frames can be estimated by applying Kalman filtering method.

\subsection{3-D reconstruction of scattering centers based on optical flow}

In case of ground based radar, the motion of a scattering center of the target in the virtual projection space [8] is equivalent to a rotation followed by a translation [4]

$$
\left[\begin{array}{c}
X^{\prime} \\
Y^{\prime} \\
Z^{\prime}
\end{array}\right]=\left[\begin{array}{l}
t_{1} \\
t_{2} \\
t_{3}
\end{array}\right]+\left[\begin{array}{l}
\omega_{1} \\
\omega_{2} \\
\omega_{3}
\end{array}\right] \times\left[\begin{array}{c}
X \\
Y \\
Z
\end{array}\right]
$$

In the virtual projection space, the image-space coordinates of a CICSC are related with the corresponding scattering center coordinates by the following equation:

$$
\left\{\begin{array}{l}
x=f_{x} \cdot \frac{X}{Z} \\
y=f_{y} \cdot \frac{Y}{Z}
\end{array}\right.
$$

where $(x, y)^{T}$ are the image-space coordinates of the CICSC; $f_{x}$ and $f_{y}$ are the Virtual Focal Length (VFL) [8] in abscissa and ordinate. For the ISAR image sequence, the optical flow of CICSC $(u, v)^{T}$ can be completely described by its displacement between the frames [9]

$$
\left\{\begin{array}{l}
u=d x / d t \\
v=d y / d t
\end{array}\right.
$$

Substituting (3) and (4) into (2) gives

$$
\begin{aligned}
& Z=\frac{t_{1} f_{x}-x t_{3}}{u+\left[x y \omega_{1} / f_{y}-\left(f_{x}+x^{2} / f_{y}\right) \omega_{2}+y \omega_{3} f_{x} / f_{y}\right]} \\
& =\frac{t_{2} f_{y}-y t_{3}}{v+\left[\left(f_{y}+y^{2} / f_{y}\right) \omega_{1}-x y \omega_{2} ; / f_{x}-x \omega_{3} f_{y} / f_{x}\right]}
\end{aligned}
$$

Generally, we call $Z$ as the depth of the scattering center in the virtual projection space. From (5), associating with $X$ and $Y$, the 3-D parameters of 


\section{EXPERIMENTAL IDENTIFICATION RESULTS}

The experimental identification results for 5 types of simulated targets are shown in Table 1. The 5 targets are clearly identified just with 9 group samples for each target in the aspect angle range of $\pm 30^{\circ}$, while more than 60 samples must be obtained by the method of using range profiles [2-4]. The average recognition rate is also measured to $89.5 \%$.

Table 1. Experimental identification results for 5 types of simulated targets

\begin{tabular}{llllll}
\hline Targets & A & B & C & D & E \\
\hline Recognition rate $(\%)$ & 87.5 & 92.5 & 90 & 87.5 & 90 \\
\hline
\end{tabular}

\section{CONCLUSION}

A method of extracting the 3-D characteristic of targets from ISAR images sequence of the targets is presented in the paper. And, the target's 3$D$ structure characteristic is possible to be used to identify different radar targets.

\section{REFERENCES}

1. Smith C. R., Goggans P. M.. Radar target identification. IEEE. Antenna and Propagation Magazine, 1993,35(2):23-33

2. Li H. J., Yang S. H.. Using range profiles as feature vectors to identify aerospace objects. IEEE Trans. on Aerospace and Electronics System, 1993, 29(3):741-748

3. Hudson S., Psaltis D.. Correlation filters for Aircraft identification from radar range profiles. IEEE Trans. AES, 1993 29(3):741-748

4. David V. . Machine vision. Prentice Hall, 1991

5. Haiqing $\mathrm{Wu}$, Gilles Y. Delisle. Precision tracking algorithms for ISAR imaging. IEEE Trans. Aerospace and Electronic System, 1996, 32(1):243-254

6. Toshiharu Itoh, Hachiro Sueda. Motion compensation for ISAR via centroid tracking. IEEE Trans. Aerospace and Electronic System, 1996, 32(3):1191-1197

7. B. Horn, B. Schunck. Determining optical flow. Artificial Intelligence, 1981, 17: 185-203

8. Feng Liu. Target 3-dimensional Feature Extraction Based on Radar Image Sequence Analyzing. Northwestern Polytechnical University Ph.d dissertation, 2004.

9. K. Kanatani. Geometric computation for machine vision. Oxford: Oxford Science Publications, 1993 